\title{
CARACTERÍSTICAS QUÍMICAS DE SOLO COM VITICULTURA ORGÂNICA E BIODINÂMICA
}

\author{
Luiz Ermindo Cavallet ${ }^{1}$, Michele di Foggia ${ }^{2}$ e Carine Rusin ${ }^{3}$ \\ ${ }^{1}$ Docente associado, Departamento de Ciências Biológicas, Universidade Estadual do Paraná - UNESPAR, e-mail: \\ luiz.cavallet@unespar.edu.br \\ ${ }^{2}$ Departamento de Ciências Agrárias, Alma Mater Studiorum, Universidade de Bolonha - UNIBO, e-mail: \\ micheled@chem.unibo.it \\ ${ }^{3}$ Mestranda, bolsista CAPES, Universidade Estadual do Centro Oeste - UNICENTRO, e-mail: carine.rusin@gmail.com
}

RESUMO: O sistema de cultivo biodinâmico é uma modalidade de sistema de produção orgânica em que se aplicam produtos segundo um protocolo definido, permitindo estimular algumas características tidas como benéficas para solo e planta. Objetivou-se avaliar as diferenças em valores de parâmetros de solo entre agricultura biodinâmica e orgânica em vinha (Vitis vinifera L.). Foram avaliados matéria orgânica, $\mathrm{N}$ total, $\mathrm{P}$ assimilável, $\mathrm{K}, \mathrm{Ca}, \mathrm{Mg}, \mathrm{Na}$ trocável, $\mathrm{Fe}, \mathrm{Mn}, \mathrm{Zn}, \mathrm{Cu}$ e B assimilável. Teores solúveis de $\mathrm{Cl}, \mathrm{K}, \mathrm{Ca}, \mathrm{Mg}$, e $\mathrm{Na}$; CTC; salinidade; $\mathrm{C} / \mathrm{N}$; amônio, nitrato; areia, silte e argila. Houve maior concentração de amônio no sistema biodinâmico. Não foram encontradas diferenças nos demais parâmetros de fertilidade do solo. O solo com sistema biodinâmico apresentou valores superiores de $\mathrm{Ca}$ (solúvel em água) e do índice de salinidade. É possível que essa maior quantidade do elemento cálcio tenha sido originado da constituição química dos preparados biodinâmicos.

Palavras-chave: Vitis vinifera L., nitrato, amônio, preparados biodinâmicos

\section{CHEMICAL CHARACTERISTICS OF THE SOIL IN A VINEYARD UNDER ORGANIC AND BIODYNAMIC MANAGEMENT}

\begin{abstract}
Biodynamic farming is a variation of the organic production system in which specific products are applied with a well-defined protocol. One of the main effects of these preparations should be the stimulation of beneficial soil and plant characteristics. The objective was to determine the soil parameters differences existing between biodynamically and organically growth vineyards (Vitis vinifera L.). Several parameters were evaluates: organic matter, total $\mathrm{N}, \mathrm{pH}\left(\mathrm{H}_{2} \mathrm{O}\right)$, available $\mathrm{P}$, exchangeable values of $\mathrm{K}, \mathrm{Ca}, \mathrm{Mg}$, assimilable values of $\mathrm{Fe}, \mathrm{Mn}, \mathrm{Cu}$ and $\mathrm{B}$, soluble levels of $\mathrm{Cl}, \mathrm{K}, \mathrm{Ca}, \mathrm{Mg}$ and $\mathrm{Na}, \mathrm{CTC}$, salinity, $\mathrm{C} / \mathrm{N}$, ammonium, nitrate, sand, silt and clay. There was a higher concentration of ammonium in the biodynamic system. With respect to the other soil fertility parameters there were no differences between the two types of management. In biodynamic vineyard, soil had a higher concentration of (soluble) calcium and higher value of salinity. It is possible that the increased amount of calcium in the soil had come from the chemical composition of the biodynamic preparations.
\end{abstract}

Key-words: Vitis vinifera L., nitrate, ammonium, biodynamic preparations 


\section{INTRODUÇÃO}

A agricultura orgânica tem uma história controversa e é considerada por alguns como uma abordagem ineficiente para produção de alimentos. No entanto, os alimentos e bebidas orgânicos são um segmento de mercado em rápido crescimento na indústria global de alimentação. Os sistemas de cultivo orgânico tem produtividade mais baixa em comparação com a agricultura convencional. No entanto, eles são mais rentável e ambientalmente mais sustentáveis, com alimentos tão ou mais nutritivos, contendo menos (ou nenhum) resíduo de pesticidas, quando comparado com a agricultura convencional (Reganold e Wachter, 2016).

Devido a isso, a crescente preocupação do consumidor com a poluição ambiental causado pela atividade agrícola e a qualidade do alimento em relação à saúde humana tem estimulado formas alternativas de agricultura, como a agricultura orgânica e a agricultura biodinâmica (Ponzio et al., 2013).

O que caracteristicamente diferencia ambos os sistemas é que na agricultura biodinâmica há uso obrigatório de um conjunto de preparados fermentados específicos aplicados nas plantas e/ou no solo em quantidades muito pequenas. A maioria deles proposto por Rudolf Steiner (1861-1925), sendo que para esses preparados é atribuído estimular o ciclo de nutrientes do solo, aumentar a fotossíntese e ajudar na evolução ótima do composto, aumentando a qualidade de solo e planta (Turinek et al. 2009).

Mais especificamente, em se tratando da viticultura, o manejo biodinâmico se insere como uma importante abordagem para a sustentabilidade do meio ambiente, não somente para a cultura agrícola, como também para os seus produtos derivados, como, por exemplo, o vinho (Preston, 2008; Zucca et al, 2009).

A ação dos preparados biodinâmicos sobre tecido vegetal, grão de uva e vinho já é conhecida e aparenta responder mais quando comparado com respostas de parâmetros de solo. Por exemplo, Perpinello et al. (2015), a partir de um pomar com produção orgânica convertido em biodinâmico encontraram diferençasna composição e nosatributos sensoriais do vinho, tais com quanto aobaixo teor alcoólico, acidez, intensidade de cor, teor de polifenois totais, entre outros compostos mais. Reeve et al. (2005), em um estudo de seis anos comparando esses dois 
sistemas com cultivo de viticultura, encontraram evidências de que os preparados biodinâmicos podem influenciar no aumento do volume de poda da videira, assim como do grau Brix, de fenóis totais e antocianinas totais no vinho obtido.

Com relação à agricultura orgânica em si, de um modo geral, ela demonstra ocasionar no solo valores mais elevados de carbono orgânico e biomassa microbiana quando comparada com o sistema convencional. A complexidade aumenta quando os três sistemas de produção (orgânico, biodinâmico e convencional) são estudados juntos e o carbono da biomassa microbiana e carbono orgânico possuem evidente diferença. O carbono orgânico tende a ser ligeiramente superior no sistema orgânico com relação ao sistema biodinâmico e o carbono microbiano tende a ser mais elevado na agricultura biodinâmica. De qualquer forma, ambos os sistemas apresentam valores mais elevados quando comparados com o sistema convencional (Fliessbach et al., 2007; Gattinger et al., 2012, citado por Reganold e Wachter, 2016).

Assim, ainda com relação ao aspecto fisiológico dos efeitos dos preparados biodinâmicos nas plantas, supõe-se que esses influenciam o metabolismo da biota do solo e a nutrição da planta. Por exemplo, Scheller e Raupp (2005) conduziram um experimento de longa duração comparando adubação mineral, composto orgânico de origem animal e composto orgânico de origem animal com aplicação de preparados biodinâmicos. Entre suas considerações, afirmam que as diferenças entre os tratamentos não dependem somente do tipo de aminoácidos fornecido pelo composto utilizado, mas também da variação do metabolismo da biota do solo induzida por esses aminoácidos.

Nesse sentido, quando se aborda as transformações na biota do solo em função da predominância da disponibilidade de determinados aminoácidos, cabe ressaltar o já notório conhecimento que a dinâmica do ciclo do $\mathrm{N}$ no solo induz transformações bioquímicas no processo de mineralização, a qual conduz à formação de íons solúveis como o nitrato e o amônio, os quais estão estreitamente ligados ao metabolismo microbiano do solo. Assim, é de se supor que o metabolismo é particularmente influenciado pela adoção de práticas de agricultura biodinâmica, na qual os íons nitrato e amônio podem ser utilizados como indicadores biológicos para o estudo dos efeitos dos preparados biodinâmicos. 
Por outro lado, em se tratando de características de solo e planta, os preparados biodinâmicos às vezes apresentam resultados e às vezes não o fazem, como afirmam Reeve et al. (2005) e Turinek et al. (2009). Possivelmente isso é devido à condição do solo, sendo que as respostas podem ser mais evidentes quando o solo é de baixa fertilidade.

Portanto, considerando que os preparados biodinâmicos estimulam o ciclo de nutrientes é possível que ocorram transformações químicas no solo após os mesmos serem aplicados. Assim, o objetivo do presente trabalho é avaliar as possíveis dinâmicas e alterações químicas no solo, tendo como indicadores os parâmetros comumente utilizados em análises de rotina de solo e também quanto aos íons nitrato e amônio, em um experimento de longa duração com viticultura.

\section{MATERIAIS E MÉTODOS}

\subsection{DESCRIÇÃO DA ÁREA, DELINEAMENTO EXPERIMENTAL E MANEJO DA CULTURA}

O experimento de longa duração foi conduzido desde 2008, em um vinhedo adulto implantado no ano de 2003, cv. Sangiovese (clone FEDIT 30 ESAVE), V. Vinifera L., enxertado sob Kober 5BB, junto à Estação Experimental Terra Naldi, do Centro de Pesquisa para a Produção Vegetal (CPRV), situada na localidade de Tebano, no município de Faenza, Emilia Romagna, Itália (44²17' ${ }^{\circ}$, $11^{\circ}$ 52'59"E, 117m, classificação climática de KöppenGeiger Cfa, clima subtropical úmido), em um declive médio, com orientação sudeste / noroeste. O solo utilizado é provavelmente um Cambissolo Flúvico Carbonático típico, o qual predomina na estação experimental onde foi conduzido o experimento.

As plantas foram espaçadas a 2,8 $\mathrm{m} \times 1,0 \mathrm{~m}\left(3.571\right.$ plantas $\left.\mathrm{ha}^{-1}\right)$. A partir de 2007, este vinhedo comercial foi conduzido e certificado como orgânico por parte do ICEA (Instituto de Certificação Ética e Ambiental), portanto, de acordo com o Regulamento CE 834 de 2007 (EC, 2007, citado por Botelho et al., 2015). Em 2008, a superfície total (2 ha) foi dividida em duas grandes áreas uniformes com características químicas do solo semelhante, conforme consta na Tabela 1. 
Cada área foi submetida a um método de cultivo específico (tratamentos): (1) a agricultura orgânica, conduzida de acordo com o Regulamento CE 834 de 2007 e (2) a agricultura biodinâmica, composta pela agricultura orgânica, mais as aplicações de preparados biodinâmicos de acordo com as normas Demeter (Demeter International, 2012, citado por Perpinello et al., 2015). Cada tratamento foi composto de sete parcelas (repetições), totalizando 14 unidades experimentais, cada uma com 12 videiras, totalizando 168 videiras no experimento.

Não foi feita a distribuição ao acaso das unidades experimentais devido à possibilidade de que isso poderia potencialmente causar problemas na gestão deste experimento de longa duração. Isso devido a possíveis efeitos de preparados biodinâmicos agirem além dos limites de cada parcela, tais como efeitos sobre microrganismos do solo, micorrizas fúngicas promovidas pelo acúmulo de nutrientes e troca de metabólitos.

No final de cada ciclo vegetativo da videira foram semeadas em linhas alternadas espécies herbáceas, tais como feijão fava (Vicia faba), cevada (Hordeum vulgare), trevo subterrâneo (Trifolium subterraneum) e mostarda castanha (Brassica juncea), em toda a área do experimento. A vegetação foi roçada no final da primavera (junho) e a biomassa foi mantida na superfície do solo.

O controle de pragas e doenças foi realizado com produtos orgânicos permitidos conforme legislação pertinente para esse sistema de produção, consistindo principalmente em de produtos a base de cobre (média de $6 \mathrm{~kg} \mathrm{ha}^{-1}$ ano $^{-1}$ ) e enxofre (em média de $70 \mathrm{~kg} \mathrm{ha}^{-1}$ ano $^{-1}$ ).

Desde o ano de 2007 não se utilizou irrigação ou qualquer tipo de adubação na área do experimento. No ano de 2008 realizou-se coleta de amostra de solos em cada unidade experimental na camada de 0-40 $\mathrm{cm}$ de profundidade para análise. Não foram observadas diferenças significativas nos parâmetros avaliados ( Tabela 1).

\subsection{CONDIÇÕES CLIMÁTICAS}

A estação vegetativa de 2011 foi marcada por temperaturas médias bem acima do normal para a época, com picos de temperatura de $30^{\circ} \mathrm{C}$ em agosto. A partir da brotação à colheita, a 
umidade relativa média $(\mathrm{RH})$ variou de 40 a 70\%, valores mais elevados foram observados durante a primavera (92\%) e a mais baixa (38\%) no final de agosto. O total de precipitação (204 $\mathrm{mm}$ ) a partir da brotação até a colheita foi esporádico durante a primavera e quase ausente durante o amadurecimento.

Em 2012, quando foram coletadas as amostras de solo, a temperatura média mostrou uma tendência crescente com início em março $\left(10^{\circ} \mathrm{C}\right)$ até agosto, e em seguida estabilizou em aproximadamente $30^{\circ} \mathrm{C}$, no final do mês. A média de $\mathrm{RH}$ da brotação à colheita variou de $35 \mathrm{a}$ $70 \%$, mostrando o valor mais baixo (37\%) no final de julho, enquanto os picos mais elevados ocorreram durante maio (88\%) e no início de setembro (89\%). O total de precipitação (342 $\mathrm{mm}$ ), registado a partir da brotação até a colheita, ocorreu principalmente na primavera.

Ainda com relação ao RH observou-se que nos três meses que antecederam a segunda coleta de amostras, a qual ocorreu em 18 de setembro de 2012, houve muito pouca precipitação, inclusive sendo atribuída com uma histórica estiagem, pois choveram nos meses de junho, julho e agosto somente $16 \mathrm{~mm}$. A estiagem acabou no dia 31 de agosto e posteriromente houve bastante precipitação, pois somente no mês de setembro choveu $91 \mathrm{~mm}$.

\subsection{COLETA DE AMOSTRAS DE SOLO}

Além da amostragem de solos realizada no início do experimento em 2008, já descrita acima e apresentadas abaixo na Tabela 1 por Botelho et al. (2015), foram realizadas durante o ano de 2012 duas coletas de amostras de solos, sendo a primeira na primavera, em maio, durante a fase de florescimento e a segunda no outono, em setembro, na fase de maturação.

Tanto na primeira como na segunda coleta de solos, foram amostradas a camada superficial $(0-20 \mathrm{~cm})$ e a camada profunda $(20-40 \mathrm{~cm})$. As determinações de parâmetros de rotina de solos foram feitas somente na camada superficial com amostras somente da primeira coleta de solos (maio), totalizando assim 14 amostras para essa avaliação, com sete repetições em apenas uma camada de solo. Já para as determinações de nitrato e amônio foram feitas com ambas as coletas (maio e setembro) em ambas as camadas, totalizando 28 amostras, com sete repetições em duas camadas de solo. 
Tabela 1. Valores de análises de solo em sistemas de produção orgânico (ORG) e biodinâmico (BIO), no início do experimento (2008) cultivado com viticultura, na camada superficial $(0-20 \mathrm{~cm})$.

\begin{tabular}{|c|c|c|}
\hline Parâmetro & ORG & $\mathrm{BIO}$ \\
\hline $\mathrm{pH}\left(\mathrm{H}_{2} \mathrm{O}\right)$ & 8,14 n.s. & 8,05 \\
\hline Mat. Org. (\%) & 1,9 n.s. & 1,7 \\
\hline $\mathrm{N}$ total $(\%)$ & 1,32 n.s. & 1,41 \\
\hline $\mathrm{P}$ assim. $\left(\mathrm{mg} \mathrm{kg}^{-1}\right)$ & 33,43 n.s. & 27,14 \\
\hline $\mathrm{K}$ troc. $\left(\mathrm{mg} \mathrm{kg}^{-1}\right)$ & 248 n.s. & 289 \\
\hline Ca troc. $\left(\mathrm{mg} \mathrm{kg}^{-1}\right)$ & 3763 n.s. & 3634 \\
\hline $\mathrm{Mg}$ troc. $\left(\mathrm{mg} \mathrm{kg}^{-1}\right)$ & 311 n.s. & 277 \\
\hline Na troc. $\left(\mathrm{mg} \mathrm{kg}^{-1}\right)$ & 46,57 n.s. & 44,43 \\
\hline $\mathrm{Fe}$ assim. $\left(\mathrm{mg} \mathrm{kg}^{-1}\right)$ & 19,49 n.s. & 18,35 \\
\hline Mn assim. $\left(\mathrm{mg} \mathrm{kg}^{-1}\right)$ & 7,55 n.s. & 7,01 \\
\hline $\mathrm{Zn}$ assim. $\left(\mathrm{mg} \mathrm{kg}^{-1}\right)$ & 1,92 n.s. & 2,08 \\
\hline $\mathrm{Cu}$ assim. $\left(\mathrm{mg} \mathrm{kg}^{-1}\right)$ & 12,97 n.s. & 16,27 \\
\hline B assim. (mg kg $\left.{ }^{-1}\right)$ & 0,48 n.s. & 0,45 \\
\hline CTC (meq 100g $\mathrm{g}^{-1}$ ) & 22,1 n.s. & 21,2 \\
\hline $\mathrm{Cl}$ dispon. (mg kg-1) & n.d. & \\
\hline $\mathrm{K}$ dispon. (mg kg-1) & n.d. & \\
\hline $\mathrm{Ca}$ dispon. $\left(\mathrm{mg} \mathrm{kg}^{-1}\right)$ & n.d. & \\
\hline $\mathrm{Mg}$ dispon. (mg kg $\left.{ }^{-1}\right)$ & n.d. & \\
\hline $\mathrm{Na}$ dispon. $\left(\mathrm{mg} \mathrm{kg}^{-1}\right)$ & n.d. & \\
\hline Salinidade $\left(\mathrm{mS} \mathrm{cm}^{-1}\right)$ & n.d. & \\
\hline $\mathrm{C} / \mathrm{N}$ & n.d. & \\
\hline Areia \% & n.d. & \\
\hline Silte $\%$ & n.d. & \\
\hline Argila $\%$ & n.d. & \\
\hline
\end{tabular}

Botelho et al. (2015); n.s., não significativo (P $\leq 0,05)$; n.d., não determinado

A primeira coleta de solos foi realizada no dia 14 de maio de 2012, sendo que para a camada superficial $(0-20 \mathrm{~cm})$ foi utilizada uma pá de corte ao invés de um trado holandês, pois se pretendeu que o material das amostras não sofresse demasiada deformação, uma vez que em algumas das amostras seriam também verificados parâmetros de morfologia de raiz por parte de um experimento concomitante. Para a coleta na segunda camada $(20-40 \mathrm{~cm})$ foi utilizado um trado Calador, uma vez que se observou forte compactação do solo na camada 20-40 cm e isso devido ao solo estar muito ressequido. Já para a segunda amostragem de solos em setembro, 
a coleta de amostras foi feita com auxilio de um trado holandês. Após a coleta, as amostras foram conduzidas ao resfriamento ininterrupto a- $4^{\circ} \mathrm{C}$.

As análises de solo foram realizadas segundo manual para Metodologia Oficial para Análise Química de Solos na Itália (Ministero Delle Politiche Agricole e Forestali, 1999) da forma como é descrita a seguir: $\mathrm{pH}$ em água por determinação potenciométrica; matéria orgânica seguindo o método de Walkley-Black; N total por Kjeldahl; P assimilável; K, Na, Ca e Mg trocáveis e CTC com cloreto de bário/acetato de amônio; Fe, Mn, Zn, Cu assimilável, DTPA; B assimilável por extração com água quente; textura pelo método de Bouyoucos; nitrato e amônio através da extração com $\mathrm{KCl}$.

O delineamento experimental adotado foi blocos ao acaso e a análise estatística dos dados foi feita utilizando o sistema computacional SAS (SAS Insitute, Cary, NC) utilizando o teste de Student-Newman-Keuls $(\mathrm{p}<0,05)$.

\subsection{PREPARADOS BIODINÂMICOS}

Os preparados biodinâmicos foram adquiridos e elaborados segundo o padrão Demeter (Demeter International, 2012, citado por Perpinello et al, 2015) e a aqueles utilizados nesse experimento, seguem abaixo com o respectivo local de aplicação e quantidade desde o início do experimento: Fladen (900 $\left.\mathrm{g} \mathrm{ha}^{-1}\right)$, aplicação no solo; 500K (600 $\left.\mathrm{g} \mathrm{ha}^{-1}\right)$ aplicação no solo; 501 (50 $\left.\mathrm{g} \mathrm{ha}^{-1}\right)$, aplicação foliar de pó de quartzo finamente moído (Spaccini et al, 2012); 500 $\left(800 \mathrm{~g} \mathrm{ha}^{-1}\right)$, aplicação no solo. A quantidade total de preparados aplicados somam, portanto, $2.350 \mathrm{~g} \mathrm{ha}^{-1}$. As aplicações dos mesmos nas parcelas do tratamento biodinâmico foram feitas através de pulverização, cujo respectivo conteúdo foi diluindo em $30 \mathrm{~L} \mathrm{ha}^{-1}$ de água. Além dos preparados biodinâmicos, em 2010, foram aplicados no caule das plantas desse tratamento 130 $\mathrm{kg} \mathrm{ha}^{-1}$ de pasta de tronco, uma mistura de esterco fresco bovino, infusão de urtiga e erva cavalinha, areia, bentonita e água.

A constituição de cada preparado é descrita por Botelho et al. (2015), conforme análises laboratoriais realizadas com os produtos adquiridos e aplicados no mesmo experimento. Observa-se que os preparados 500, 500K e Fladen possuem em suas constituições de 17,4 a 
$19,6 \%$ de carbono total; de 1,6 a $1,9 \%$ de $\mathrm{N}$ total; 0,43 a $0,59 \%$ de $\mathrm{P} ; 0,64$ a $0,92 \%$ de $\mathrm{K} ; 3,10$ a $5,03 \%$ de $\mathrm{Ca} ; 0,66$ a $1,15 \%$ de $\mathrm{Mg} ; 0,06$ a $0,29 \%$ de $\mathrm{Na}$ e 0,28 a 0,35 de S. O preparado 501 possui concentrações desses parâmetros muito abaixo dos primeiros, excetuando-se o $\mathrm{N}$ total que é da ordem de $1,38 \%$.

\section{RESULTADOS E DISCUSSÃO}

\subsection{ANÁLISES DE FERTILIDADE DO SOLO}

Os resultados de análise de rotina da camada superficial do solo $(0-20 \mathrm{~cm})$ são descritos na Tabela 2. Excetuando-se o elemento cálcio disponível e a salinidade, com relação às características químicas não foram observadas diferenças entre os dois tratamentos. 
Tabela 2. Análises de solo em sistema de produção orgânico (ORG) e biodinâmico (BIO) cultivado com viticultura, na camada superficial $(0-20 \mathrm{~cm})$. Média de sete repetições

\begin{tabular}{|c|c|c|}
\hline Parâmetro & ORG & $\mathrm{BIO}$ \\
\hline$\overline{\mathrm{pH}\left(\mathrm{H}_{2} \mathrm{O}\right)}$ & 7,48 n.s & 7,42 \\
\hline Mat. Org. (\%) & 3,34 n.s & 3,57 \\
\hline $\mathrm{N}$ total $(\% \mathrm{o})$ & $2,31 \mathrm{n} . \mathrm{s}$ & 2,45 \\
\hline $\mathrm{P} \operatorname{assim}\left(\mathrm{mg} \mathrm{kg}^{-1}\right)$ & 27,43 n.s & 32,28 \\
\hline $\mathrm{K}$ troc $\left(\mathrm{mg} \mathrm{kg}^{-1}\right)$ & 304 n.s & 324 \\
\hline Ca troc. $\left(\mathrm{mg} \mathrm{kg}^{-1}\right)$ & 3902 n.s & 3900 \\
\hline $\mathrm{Mg}$ troc. $\left(\mathrm{mg} \mathrm{kg}^{-1}\right)$ & $176 \mathrm{n} . \mathrm{s}$ & 169 \\
\hline $\mathrm{Na}$ troc. $\left(\mathrm{mg} \mathrm{kg}^{-1}\right)$ & 44,00 n.s & 38,43 \\
\hline $\mathrm{Fe} \operatorname{assim}\left(\mathrm{mg} \mathrm{kg}^{-1}\right)$ & 18,18 n.s & 20,35 \\
\hline Mn assim. $\left(\mathrm{mg} \mathrm{kg}^{-1}\right)$ & 50,19 n.s & 53,47 \\
\hline $\mathrm{Zn}$ assim. $\left(\mathrm{mg} \mathrm{kg}^{-1}\right)$ & 2,84 n.s & 3,37 \\
\hline $\mathrm{Cu}$ assim. $\left(\mathrm{mg} \mathrm{kg}^{-1}\right)$ & 43,91 n.s & 48,48 \\
\hline B assim. $\left(\mathrm{mg} \mathrm{kg}^{-1}\right)$ & $0,70 \mathrm{n} . \mathrm{s}$ & 0,72 \\
\hline CTC (meq 100g-1) & 21,89 n.s & 21,85 \\
\hline $\mathrm{Cl}$ dispon. ( $\left.\mathrm{mg} \mathrm{kg}^{-1}\right)$ & 21,28 n.s & 19,85 \\
\hline $\mathrm{K}$ dispon. $\left(\mathrm{mg} \mathrm{kg}^{-1}\right)$ & 36,94 n.s & 45,07 \\
\hline Ca dispon. $\left(\mathrm{mg} \mathrm{kg}^{-1}\right)$ & $194 \mathrm{~b}$ & $224 \mathrm{a}$ \\
\hline $\mathrm{Mg}$ dispon. $\left(\mathrm{mg} \mathrm{kg}^{-1}\right)$ & $14 \mathrm{n} . \mathrm{s}$ & 16 \\
\hline $\mathrm{Na}$ dispon. $\left(\mathrm{mg} \mathrm{kg}^{-1}\right)$ & 8,24 n.s & 8,50 \\
\hline Salinidade $\left(\mathrm{mS} \mathrm{cm}^{-1}\right)$ & $0,42 \mathrm{~b}$ & $0,47 \mathrm{a}$ \\
\hline $\mathrm{C} / \mathrm{N}$ & $8,40 \mathrm{n} . \mathrm{s}$ & 8,46 \\
\hline Areia \% & $29,71 \mathrm{n} . \mathrm{s}$ & 33,00 \\
\hline Silte\% & 44,85 n.s & 42,57 \\
\hline Argila \% & 25,42 n.s & 24,42 \\
\hline
\end{tabular}

Por outro lado, se observa que o solo apresenta boas condições de fertilidade, com altas concentrações de macronutrientes. Pois, com relação aos tratamentos orgânico e biodinâmico respectivamente, tem-se: matéria orgânica (3,34 e 3,57\%), $\mathrm{N}$ total (2,31 e 2,45 \%o), K trocável (304 e $324 \mathrm{mg} \mathrm{kg}^{-1}$ ) e P assimilável (27,43 e 32,28 $\mathrm{mg} \mathrm{kg}^{-1}$ ), Ca trocável (3.901 e 3.900 mg kg${ }^{1}$ ) e $\mathrm{Mg}$ trocável (166 e $179 \mathrm{mg} \mathrm{kg}^{-1}$ ), sendo que o $\mathrm{pH}\left(\mathrm{em} \mathrm{H}_{2} \mathrm{O}\right)$ é considerado com sub-alcalino (7,48 e 7,42), indicando, portanto ausência de acidez. 
Dessa forma, o solo pode ser considerado de boa fertilidade e essa condição pode ter mascarado o efeito dos preparados biodinâmicos sobre os teores de nutrientes, como sugerem Reeve et al. (2005) e Turinek et al. (2009). Raupp e König (1996), de forma similar, afirmam que os preparados biodinâmicos demonstram possuir maior efeito em condições de solos com baixa fertilidade, enquanto em solos mais férteis os efeitos dos mesmos são pouco evidentes.

Por outro lado, observa-se que os preparados biodinâmicos causaram aumento do índice de salinidade, apesar do solo utilizado já se encontrar dentro dos limites tidos como médio a alto $\left(0,42\right.$, e $\left.0,47 \mathrm{mS} \mathrm{cm}^{-1}\right)$ com relação a esse parâmetro. Provavelmente esse efeito tenha ocorrido com a contribuição do elemento cálcio, que é um componente da salinidade. O mesmo, na sua forma disponível, apresentou significativa concentração no tratamento biodinâmico, onde teve valor muito alto $\left(224 \mathrm{mg} \mathrm{kg}^{-1}\right)$, diferenciando-se do tratamento orgânico, o qual teve valor classificado como apenas alto $\left(194 \mathrm{mg} \mathrm{kg}^{-1}\right)$.

Assim, sugere-se que a aplicação dos preparados biodinâmicos tenha causado maior aumento e solubilização do elemento cálcio, de onde se pode supor que o aumento da salinidade pode ser atribuído à constituição química dos preparados biodinâmicos, uma vez que o cálcio disponível compõe o índice de salinidade.

Tal afirmação é reforçada quando se avalia a concentração de cálcio na constituição dos preparados biodinâmicos com relação à quantidade total aplicada. Assim, considerando apenas nos preparados 500, 500K e Fladen, os quais possuem visivelmente maior concentração de cálcio $(3,10$ a $5,03 \%)$, ao final a quantidade total de cálcio aplicada no experimento será aproximadamente de 68 a 110 g. Portanto, como na constituição dos preparados há significativa concentração de cálcio, é possível que os mesmos tenham sido a origem da maior concentração desse elemento no tratamento biodinâmico e isso possa ter influenciado também nos maiores valores de salinidade.

Com relação à matéria orgânica Fliessbach et al. (2007) verificaram diminuição dessa no sistema de produção biodinâmico e no presente experimento a mesma permaneceu inalterada. Porém, no presente caso o solo utilizado tinha uma quantidade alta da mesma, em média 3,46\%, e essa condição pode ter impedido a ação dos preparados biodinâmicos. Além disso, a condução do experimento teve um manejo de plantas de cobertura que contribuiu para repor eventual 
diminuição de matéria orgânica no terreno. Pois, no final de cada ciclo vegetativo da videira foram semeadas em linhas alternadas espécies herbáceas e essa vegetação foi roçada no final da primavera (junho) e a biomassa foi mantida na superfície do solo.

Adicionalmente, observa-se novamente que o fato de não se ter ocorrido alterações na constituição da matéria orgânica entre os dois sistemas é condizente com que apresentam Reeve et al. (2005) e Turinek et al. (2009), quando afirmam que o os preparados biodinâmicos às vezes apresentam resultados e às vezes não. Possivelmente isso é devido à condição do solo, sendo que as respostas são mais evidentes quando o solo é de baixa fertilidade, o que não ocorreu no presente caso.

\subsection{NITRATO E AMÔNIO}

Além dos parâmetros químicos de análise de rotina de solo também foram estudados a concentração dos íons nitrato e amônio em duas camadas diferentes de solo (0-20 cm e 20-40 $\mathrm{cm}$ ), em dois momentos diferentes, sendo a primeira em maio, durante a primavera, e a outra em setembro, no início do outono, cujos resultados encontram-se na Tabela 3.

Tabela 3. Concentração de amônio $\left(\mathrm{NH}_{4}{ }^{+}\right)$e nitrato $\left(\mathrm{NO}_{3}{ }^{-}\right)$no solo com produção orgânica $(\mathrm{ORG})$ e biodinâmica $(\mathrm{BIO})$ em duas profundidades $(0-20$ e 20-40 cm). Coleta de amostra realizada nos meses de maio e setembro de 2012. Média de 7 repetições.

\begin{tabular}{|c|c|c|c|c|}
\hline & \multicolumn{2}{|c|}{ Amostragem em maio } & \multicolumn{2}{|c|}{ Amostragem em setembro } \\
\hline & $\begin{array}{c}\mathrm{NH}_{4}{ }^{+} \\
\left(\mathrm{mg} \mathrm{L}^{-1}\right)\end{array}$ & $\begin{array}{c}\mathrm{NO}_{3}^{-} \\
\left(\mathrm{mg} \mathrm{L}^{-1}\right)\end{array}$ & $\begin{array}{c}\mathrm{NH}_{4}^{+} \\
\left(\mathrm{mg} \mathrm{L}^{-1}\right)\end{array}$ & $\begin{array}{c}\mathrm{NO}_{3}^{-} \\
\left(\mathrm{mg} \mathrm{L}^{-1}\right)\end{array}$ \\
\hline Camada solo (CS) & n.s. & $* *$ & n.s. & $* *$ \\
\hline Tratamento $(T R)$ & $* *$ & n.s. & n.s. & n.s. \\
\hline Interação (CS $x T R)$ & n.s. & $* *$ & n.s. & n.s. \\
\hline \multicolumn{5}{|l|}{ Tratamento } \\
\hline$(0-20 \mathrm{~cm})$ ORG & $8,25 \mathrm{ab}$ & $4,59 \mathrm{~b}$ & 7,48 & $3,00 \mathrm{a}$ \\
\hline$(20-40 \mathrm{~cm}) \mathrm{ORG}$ & $5,96 \mathrm{~b}$ & $4,61 \mathrm{~b}$ & 7,11 & $1,74 \mathrm{~b}$ \\
\hline$(0-20 \mathrm{~cm}) \mathrm{BIO}$ & $8,90 \mathrm{ab}$ & $4,36 \mathrm{~b}$ & 8,65 & $4,64 \mathrm{a}$ \\
\hline$(20-40 \mathrm{~cm}) \mathrm{BIO}$ & $9,61 \mathrm{a}$ & $6,58 \mathrm{a}$ & 7,84 & $1,84 \mathrm{~b}$ \\
\hline
\end{tabular}

Valores seguidos da mesma letra na coluna não diferenciam $(p<0.05)$. ** $(p<0.01)$ 
Com relação ao tipo de tratamento, observa-se que na primeira coleta de amostras de solo, em maio, houve maior concentração de amônio no tratamento biodinâmico em relação ao nitrato. Maior concentração do amônio pode indicar maior atividade do processo de biodegradação da matéria orgânica do solo, portanto, com maior mobilidade de $\mathrm{N}$ nesse sistema. Isso devido a que o amônio é um intermediário molecular entre o $\mathrm{N}$ orgânico e o íon nitrato na fase de nitrificação do ciclo biogeoquímico do N. Assim, o aumento da disponibilidade de nutrientes no solo, especialmente de $\mathrm{N}$ originado do processo de biodegradação da matéria orgânica, pode ocasionar o incremento do teor total de $\mathrm{N}$ nas folhas da videira como já observaram vários autores (Brunetto et al., 2007; Mafra et al., 2011; Brunetto et al., 2012).

Com relação à origem do efeito dos preparados biodinâmicos no aumento do teor de amônio, observa-se que esses possuem em sua constituição o elemento cálcio de forma não pouco acentuada, e essa característica provavelmente tenha contribuído para aumentar a alcalinidade do solo. Ou seja, é possível que, em parte, a aplicação dos preparados teve efeito próximo do que ocorre quando se realiza a calagem do solo. Silva et al. (1994) citado por Silva e Valle (2000) observaram aumento na disponibilidade de nitrato onde houve aumento nos teores de cálcio no solo e pH próximo da neutralidade.

Portanto, o cálcio nesse caso teve ação indireta sobre a atividade microbiana do solo, no sentido de promover esse processo e ter como resultado final o aumento de amônio. Isso devido a que o amônio é um intermediário molecular entre o nitrogênio orgânico e o íon nitrato na fase de nitrificação do ciclo do N (Souza e Fernandes, 2006). Assim, houve maior quantidade de íons nitrogenados na solução do solo para esse tratamento, como já afirmam Scheller e Raupp (2005), com particular referimento aos aminoácidos.

A atividade microbiana ocorre de forma intensa no sistema orgânico de produção, como observam Freitas et al. (2011), quando relataram crescimento de 100 a $200 \%$ do carbono da biomassa microbiana em viticultura orgânica, quando comparada com a convencional. É provável que tal intensidade de atividade microbiana tenha ocorrido também no tratamento biodinâmico, mas, mesmo assim, os preparados biodinâmicos ocasionaram um valor adicional dessa, de forma que a maior disponibilidade de amônio pode ser utilizada como um indicador 
desse aumento. Isso é condizente com Fliessbach et al. (2007) quando afirmam que o carbono microbiano tende a ser mais elevado na agricultura biodinâmica.

Com relação à distribuição de amônio e nitrato nas camadas de solo, observa-se que em maio na primeira coleta de solos, o nitrato teve concentração maior no tratamento biodinâmico na camada $20-40 \mathrm{~cm}$ de profundidade. Essa constatação pode estar relacionada à movimentação desse íon no perfil do solo.

A movimentação de um nutriente no solo é resultado da ação simultânea de vários fatores e não da ação isolada de um deles. Dentre esses fatores está incluso o conteúdo de água e a da macroporosidade do solo, segundo observação de vários autores citados por Donagemma et al. (2008).

Assim, quando da primeira coleta de solos em maio, o mesmo estava visivelmente compactado, o que demonstra a sua baixa macroporosidade, e estava também com baixo conteúdo de água. Isso promoveu pouco deslocamento desse íon e o fez acumular na camada profunda do solo. Já na segunda coleta, em setembro, a macroporoside aumentou, pois, a compactação do mesmo diminuiu consideravelmente. Essa condição foi evidenciada pela possibilidade de executar a coleta de solo com um trado holandês ao invés de um trado para solos compactados, como foi utilizado na primeira coleta. Assim, o fato de o solo estar bem mais friável na segunda coleta, também indica que no mesmo havia suficiente conteúdo de água para aumentar o deslocamento desse íon no solo.

Esses dois fatores, quais sejam, o aumento da macroporosidade e o aumento do conteúdo de água no solo favoreceu o deslocamento do nitrato para mais abaixo da camada profunda (20$40 \mathrm{~cm}$ ). Segundo Andrade et al. (2009) a lixiviação do nitrato é potencializada pelas propriedades físicas dos solos, pelas práticas agrícolas adotadas e pela elevada aplicação de água. No presente caso a elevada precipitação que ocorreu dias antes da coleta de solo teve papel similar a uma elevada aplicação de água via irrigação, como afirmam os autores.

No sentido inverso, a absorção do nitrato pelas plantas da videira em sua fase de maturação auxiliou para esgotar a reserva de nitrato na camada mais profunda, onde se concentram grande parte do volume do sistema radicular da videira. O nitrogênio é absorvido e transportado pelas 
videiras na forma de nitrato, onde sofre redução para nitrito e em seguida para amônio na presença da enzima redutase do nitrato (Albuquerque et al., 2009). Considerando isso, pode-se afirmar que houve maior deslocamento de nitrato para o sistema aéreo das plantas no tratamento biodinâmico, o que é condizente com Reeve et al. (2009), quando observaram maior volume de poda para a videira cultiva sob esse sistema.

Assim, o deslocamento do nitrato no perfil do solo e a absorção do mesmo pelas plantas fez com que houvesse menor quantidade de nitrato em relação ao amônio quando da segunda coleta de solo, em setembro, em relação à primeira coleta, em maio. Isso não ocorreu com o amônio devido à característica de que ele é menos móvel no solo (Luchese et al. 2000 citado por Fey et al., 2010) devido a possuir maior adsorção eletrostática que o nitrato e também ser menos preferido que esse quando da sua absorção pelas plantas (Souza e Fernandes, 2006).

Portanto, quando comparado com o tratamento orgânico, observa-se que os preparados utilizados no cultivo biodinâmico podem ter ocasionado aumentos do íon amônio e provocado distribuição do nitrato de forma diferenciada entre as camadas superficial e profunda do solo. Observa-se também que na camada superficial desse tratamento houve aumento do elemento cálcio. É provável que esse aumento do elemento cálcio tenha aumentado não somente a salinidade do solo, como o foi verificado, mas também o tenha influenciado na maior disponibilidade do íon amônio e mobilidade do nitrato na solução do solo.

\section{CONCLUSÕES}

Houve maior concentração de amônio no tratamento biodinâmico quando da avaliação no período da primavera, no mês de maio, o que não ocorreu posteriormente no mês de setembro.

Na camada superficial $(0-20 \mathrm{~cm})$ não foi observado nenhum efeito dos preparados biodinâmicos sobre os parâmetros de fertilidade do solo tais como $\mathrm{P}, \mathrm{K}$, matéria orgânica, $\mathrm{pH}$, Mg, CTC, porém houve aumento do cálcio disponível e do índice de salinidade.

É possível que essas diferenças observadas acima sejam decorrentes da ação do cálcio presente na constituição química dos preparados biodinâmicos. 


\section{REFERÊNCIAS BIBLIOGRÁFICAS}

ALBUQUERQUE, T. C. S.; SILVA, D.J.; FARIA,C. M. B. PEREIRA, J. R. Nutrição e adubação. In: SOARES, J. M.; LEÃO, P. C. S. A vitivinicultura no Semiárido brasileiro. Brasília : Embrapa Informação Tecnológica; Petrolina: Embrapa Semiárido, p. 429-480, 2009.

ANDRADE, E. M.: AQUINO, D. N.; CRISÓSTOMO, L. A.; RODRIGUES, J. O.; LOPES, F. B. Impacto da lixiviação de nitrato e cloreto no lençol freático sob condições de cultivo irrigado. Cienc. Rural, n.39, p. 88-95, 2009.

BOTELHO, R. V.; ROBERTI, R.; TESSARIN, P., GARCIA-MINA, J. M.; ROMBOLÀ, A. D. Physiological responses of grapevines to biodynamic management. Renewable Agriculture and Food Systems, p.1-12, 2015. doi:10.1017/S1742170515000320.

BRUNETTO, G.; CERETTA, C. A.; KAMINSKI, J.; MELO, G. W. B.; LOURENZI, C. R.; FURLANETTO, V.; MORAES, A. Aplicação de nitrogênio em videiras na Campanha Gaúcha: Produtividade e características químicas do mosto da uva. Ciência Rural, v.37, n.2, p.389-393, 2007.

BRUNETTO, G.; TRENTIN, G.; CERETTA, C. A.; GIROTTO, E.; LORENSINI, F.; MIOTTO, A.; MOSER, G; MELO, G. W. B. Use of the SPAD-502 in estimating nitrogen content in leaves and grape yield in grapevines in soils with different texture. American Journal of Plant Sciences, v.3, p.1.546-1.561, 2012.

DONAGEMMA, G. K.; RUIZ, H. A.; ALVAREZ, V. H.; FERREIRA, P. A.; CANTARUTTI, R. B.; SILVA, A. T. da; FIGUEIREDO, G. C. Distribuição do amônio, nitrato, potássio e fósforo em colunas de latossolos fertirrigadas. R. Bras. Ci. Solo, n.32, p. 2493-2504, 2008.

FEY, R.; ZOZ, T.; STEINER, F.; RICHART, A.; BRITO, O. R. Leaching of nitrogen in column in regarding soil particle size. Scientia Agraria, v. 11, n. 2, p. 181-185, 2010.

FLIEßBACH, A., OBERHOLZER, H. R., GUNST, L., MÄDER, P. Soil organic matter and biological soil quality indicators after 21 years of organic and conventional farming. Agriculture, Ecosystems and Environment, n. 118, p. 273-284, 2007. 
FREITAS, N.; YANO-MELO, A. da; SILVA, F. de; MELO, N.; MAIA L. Soil biochemistry and microbial activity in vineyards under conventional and organic management at Northeast Brazil. Scientia Agricola, n. 68, p. 223-229, 2011.

MAFRA, M. S. H.; CASSOL, P. C.; MIQUELLUTI, D. J.; ERNANI, P. R.; GATIBONI, L. C.; FERREIRA, E. Z.; BARROS, M.; ZALAMENA, J.; GROHSKOPF, M. A. Atributos químicos do solo e estado nutricional de videira Cabernet Sauvignon (Vitis vinifera L.) na Serra Catarinense. Revista de Ciências Agroveterinárias, v. 10, n. 1, p. 44-53, 2011.

MINISTERO DELLE POLITICHE AGRICOLE E FORESTALI. 1999. Approvazione dei Metodi ufficiali di analisi chimica del suolo. Roma. Gazzetta Ufficiale della Repubblica Italiana. Supplemento ordinario n. 248 del 21 ottobre 1999. Disponível em: http://ctntes.arpa.piemonte.it/Bonifiche/Documenti/Norme/13_Set_99.pdf acesso em $\underline{04 / 04 / 2016 .}$

PERPINEllo, G. P.; ROMBOLÀ, A. D.; SIMONI, M.; VERSARIA, A. Chemical and sensory characterization of Sangiovese red wines: Comparison between biodynamic and organic management. Food and Chenistry, v. 167, p. 145-152, 2015.

PONZIO, C.; GANGATHARAN, R.; NERI, D. Organicand biodynamic agriculture: A review in relation to sustainability. International Journal of Plant \& Soil Science, n. 2, v. 1, p. 95-110, 2013.

PRESTON, D. Viticulture and winemaking in contemporary rural change:Experience from southern France and eastern Australia. Journal of Wine Research, v. 3, n. 19, p. 159-173, 2008.

RAUPP, J.; KÖNIG, U. J. Biodynamic preparations cause opposite yield effects depending upon yield levels. Biol. Agric. Hortic, n. 13, p. 175-188, 1996.

REEVE, J. R.; CARPENTER-BOGGS, L.; REGANOLD, J. P.; YORK, A. L.; MCGOURTY, G.; MCCLOSKEY, L. P. Soil and winegrape quality in biodynamically and organically managed vineyards. American Journal of Enology and Viticulture, n. 56, p. 367-376, 2005. 
REGANOLD. J. P.; WACHTER, J. M. Organic agriculture in the twenty-first century. Nature Plants, n. 15221, v. 2, p. 1-8, 2016.

SCHELLER, E.; RAUPP, J. Amino acid and soil organic matter content of topsoil in a long term trial with farmyard manure and mineral fertilizers. Biological Agriculture and Horticulture, n. 2, p. 379-397, 2005.

SILVA, C. A.; VALE, F. R. do. Disponibilidade de nitrato em solos brasileiros sob efeito da calagem e de fontes e doses de nitrogênio. Pesq. Agropec. Bras., v. 35, n. 12, p. 2461-2471, 2000. http://dx.doi.org/10.1590/S0100-204X2000001200017.

SOUZA, S. R.; FERNANDES, M. S. Nitrogênio. In: FERNADES, M. S. Nutrição mineral de plantas. Viçosa: Sociedade Brasileira de Ciência do Solo, p. 215-252, 2006.

SPACCINI, R.; MAZZEI, P.; SQUARTINI, A.; GIANNATTASIO, M.; PICCOLO, A. Molecular properties of a fermented manure preparation used as field spray in biodynamic agriculture. Environmental Science and Pollution Research, n. 2219, p. 4214 - 4225, 2012.

TURINEK, M.; GROBELNIK-MLAKAR, S.; BAVEC, M.; BAVEC, F. Biodynamic agriculture research progress and priorities. Renewable Agriculture and Food Systems, n. 24, v. 2, p. 146-154, 2009.

ZUCCA, G.; SMITH, D. E.; MITRY, D. J. Sustainable viticulture and winerypractices in California: What is it, and do customers care? International Journal of Wine Research, n. 2, 189-194, 2009. 